\title{
EL ESPACIO AUDIOVISUAL SALVADOREÑO Y SU IMPACTO EN LA ESTRUCTURA SOCIAL. UNA PERSPECTIVA DESDE CENTROAMÉRICA
}

\author{
THE SALVADORAN AUDIOVISUAL SPACE AND ITS IMPACT ON THE \\ SOCIAL STRUCTURE. A PERSPECTIVE FROM CENTRAL AMERICA
}

Recibido: 11/08/2017 - Aceptado: 04/01/2018

\begin{abstract}
Resumen
Este artículo explora los estudios de economía política de la comunicación en Centroamérica acerca de las élites económicas que estructuran un nuevo escenario global del sistema de medios en la región. El caso salvadoreño pretende ejemplificar una metodología de relacionar la esfera mediática concentrada y centralizada en los municipios con alta actividad económica, partiendo de la hipótesis que la radio y la televisión hegemónica en El Salvador reproducen las relaciones de clase en los territorios, mientras los medios de comunicación comunitarios emergen en zonas de menor actividad económica. Posteriormente, se propone ampliar el análisis estudiando los contextos y procesos estructurados socialmente en donde se insertan las formas simbólicas para abordar una nueva estructura social y cultural en la región.
\end{abstract}

Palabras clave: Medio de comunicación, poder, concentración, estructura social, economía política, El Salvador, Centroamérica, medios comunitarios.

\begin{abstract}
This article explores the studies of politic economic of the communication in Central America about economic elites that structure a new global scenario of the media system in the region. The Salvadoran case pretend illustrate a methodology to relate the media sphere concrete and centralized in the town with high economic activity, on the basic of the hypotheses of radio and television hegemonic in El Salvador reproduces class relations in the territories, while community media emerge in areas of less economic activity. Later, it is proposed to extend the analysis by studying the socially structured contexts and processes where the symbolic forms are inserted to board a new social and cultural structure in the region.
\end{abstract}

Keywords: Mass media, power, concentration, social structure, political economy, El Salvador, Central America, community media. 


\section{Estudios de economía política sobre las élites en Centroamérica}

Desde finales de los años ochenta, la región centroamericana ha experimentado cambios significativos en cuanto al modelo económico que proyecta hacia la integración económica global, impulsadas por las políticas de ajuste estructural emanadas desde el consenso de Washington. La estrategia de modernización del Estado se fundamentaba en tres lineamientos: reestructurar la burocracia estatal y hacerla más eficiente y con menos costos; trasladar servicios públicos a manos privadas; y crear marcos regulatorios que fomentasen la inversión privada (Moreno).

En la década de los sesenta las políticas de desarrollo configuraron unas élites económicas nacionales dependientes de las empresas transnacionales subsidiarias del desarrollo nacional a través de un "Estado empresarial" que garantizaba la articulación con las empresas monopólicas internacionales (Cardoso F. H.; Faletto, E.). Años después de su fracaso como modelo, el ansiado desarrollo nacional se ha convertido en una "quimera".

Desde los años noventa, con el surgimiento de las nuevas economías vinculadas con las TIC en la "aldea global", las élites ${ }^{1}$ de la región han reconvertido sus estrategias económicas y han redefinido su relación con el Estado. Es decir, una reducción significativa de este que responde a la lógica de acumulación global. William Robinson se inclina por analizar el fenómeno de las clases dominantes regionales a partir del concepto "tecnopols" (Tecnócratas y políticos) "intelectuales orgánicos carismáticos que imponen la racionalidad capitalista de la economía global" (Robinson 437). Y prosigue que esta forma de reconfiguración de las élites o fracciones transnacionales han sido respaldadas por gobiernos de corte neoliberal simbolizados por la administración “Cristiani en El Salvador, de Calderón en Costa Rica, de Lacayo como de Chamorro en Nicaragua, de Callejas en Honduras y de Arzú en Guatemala".

Benedicte Bull considera la influencia de las "élites fuertes" en la configuración de redes de apoyo que son capaces de penetrar en lo que Garay Salcedo y León denominan "la reconfiguración cooptada del Estado" y explica que es "el proceso por el que las instituciones son manipuladas desde el interior" (Bull 120). Considera que, en el Triángulo Norte, la profundización en redes de élites y su control estatal, así como su competencia por dicho control, se ve reflejada en cuatro categorías de recursos: el dinero, los medios de coacción, la información y las ideas e ideologías, incluida la religión (Bull 117).

Como resultado, se reconfigura una influencia económica, social y política tendiente a una integración global de los grupos de poder económico. Por un lado, se mantiene su influencia a través de su "derecho de picaporte"2 al interior de los Estados que "proviene del enorme poderío económico de estos grupos; de su rol central en el financiamiento de las campañas políticas de los partidos en el poder; y de su influencia sobre los medios de comunicación social" (Segovia 91); y, por el otro, agrega, estos grupos han utilizado los medios para imponer sus agendas particulares con temas de interés 
nacional, particularmente sobre las nuevas políticas económicas neoliberales para la aprobación de tratados de libre comercio y la privatización de activos del Estado.

Se infiere que, en esa reconfiguración de las élites de poder, han comprendido la enorme influencia del sistema de medios dentro de la industria cultural para la construcción de sentido en beneficio de la reproducción de un proyecto económico. Segovia hace un llamado a pensar en los medios de infocomunicación en función del nuevo modelo económico. Por tanto, cabe preguntarse en este artículo: ¿cómo los medios de comunicación y el sistema de medios refuerzan los desafíos de la clase existente e influencian las relaciones sociales? Para tal efecto, se propone abordar la configuración y reconfiguración de la estructura del sistema de medios y los vestigios sociales resultados de esa profundización de la brecha de clases entre el sector de la comunicación privada y el sector ciudadano de la comunicación.

\section{Los estudios de economía política de la comunicación}

Existen pocos estudios sobre economía política en Latinoamérica y, en la región centroamericana es aún más considerable. Como consecuencia no existen datos relevantes para conocer el comportamiento de "la interacción entre la política actual y los procesos económicos" (Bull 2). El panorama para la economía política de la comunicación es aún más débil en cuanto develan la estructura de propiedad tendiente a la injusticia social a través de "las relaciones sociales, particularmente las relaciones de poder, que constituyen la producción, la distribución y el consumo de bienes simbólicos (Bolaño C.; Mastrini G. 45).

Sin embargo, pensar el mundo desde la racionalidad económica no debe ser solo una visión estructural del mercado y sus relaciones de poder. Mosco considera que repensar la economía política significa enfatizar el cambio social, el proceso social y las relaciones sociales, que conduzca a un análisis del proceso social y no solo de las estructuras y las instituciones.

Al observar esos procesos sociales en la región centroamericana se puede constatar que existe un consenso en cuanto a la ausencia de políticas públicas de comunicación, PPC, que contribuyan a superar las desigualdades en el acceso al sector de la infocomunicación. Un informe regional sobre marcos regulatorios de la Radiodifusión en Centroamérica concluye que los marcos jurídicos de los países de la región han facilitado "que los entes públicos de los poderes ejecutivos [actúen] con escasa independencia, nula participación ciudadana y bastante discrecionalidad" (Aler y Amarc 6). El informe se refiere a la coincidencia de las nuevas normativas en telecomunicaciones surgidas en los años noventa dentro de las políticas de ajuste estructural de desregulación de las economías. Pese a reconocer el carácter público del espectro radioeléctrico, no combatieron la concentración de frecuencias, los oligopolios de radio y televisión y no superaron el discurso del espectro saturado para generar espacios al tercer sector ciudadano de la comunicación. 
Tabla 1

APROBACIÓN DE NORMATIVAS EN CENTROAMÉRICA

\begin{tabular}{lll}
\hline Año & País & Legislación \\
\hline 1996 & Guatemala & Ley de Telecomunicaciones y Superintendencia de Comunicaciones (SIT) \\
1997 & El Salvador & Ley de Telecomunicaciones y Superintendencia de Comunicaciones (SIGET) \\
1995 & Honduras & Ley Marco del Sector de Telecomunicaciones \\
1995 & Nicaragua & Ley general de Telecomunicaciones y Servicios postales \\
1954 & Costa Rica & Ley de Radio \\
2004 & & Reglamento de Radiocomunicaciones \\
\hline
\end{tabular}

Fuente: Elaboración propia a partir de ALER/AMARC.

Al revisar la estructura económica en los mapas de medios o cartografías mediáticas que demuestran la concentración de medios en la región, es posible percatarse de que son limitados. Entre los estudios se encuentran: Rockwell, R. y Norenne, J., quienes analizan la concentración desde la capacidad de influencia de poder mediático. Otro estudio de la región es el libro Los monopolios de la verdad (Mastrini G; Becerra M.), se trata de la primera aproximación del estudio comparado del mercado de la infocomunicación para comprender la relación de la concentración de mercado por parte de élites locales o internacionales; sin embargo, los resultados de sus investigadores expresan la falta de información para complementar datos que conduzcan a medir los índices de concentración de mercado y audiencia, entre otras variables.

Pese a ello, los datos obtenidos permitieron concluir que la estructura social reproduce en la región las desigualdades sociales tendientes a generalizar la pobreza en cuanto al consumo de bienes de la industria cultural. Se ve limitada la posibilidad de acceder a los medios que no implican pago directo e incluso la población tiene problemas para acceder a los aparatos reproductores. Y desde la diversidad de medios y acceso concluyen que "Los datos aportados en la presente investigación contribuyen a sostener la tesis de quienes piensan que el sistema de medios actual dista de ser pluralista" (Mastrini G y Becerra M. 241).

Para entender esa falta de pluralidad del sistema de medios que afecta la calidad de la democracia, el libro Poder y medios en Centro América hace una aproximación sobre las políticas públicas de comunicación, a fin de incorporar a la ciudadanía como tercer sector y lograr diversificar la opinión y la producción de sentido. El estudio fue realizado en cuatro países de la región: El Salvador, Guatemala, Honduras y Nicaragua. El planteamiento del libro está influenciado de la Organización de las Naciones Unidas para la Educación y la Cultura (Unesco), que desde los años setenta se ha 
pronunciado por las políticas públicas de comunicación. El estudio retoma el enfoque de derecho de los indicadores de desarrollo mediáticos (Unesco) en congruencia con las resoluciones de la Corte Interamericana de Derechos Humanos y la Relatoría de Libertad de Expresión que consideran la comunicación como un derecho humano.

Los cuatro países coinciden en que es necesario que las naciones legislen con una política que reconozca los tres sectores de la comunicación: público, privado y ciudadano. Señalan la necesidad de regular para combatir la concentración de medios a través de la explotación del espectro radioeléctrico de la radio y la televisión como bien público y contribuir al cumplimiento del derecho humano para "fundar o utilizar cualquier medio apropiado para difundir el pensamiento y hacerlo llegar al mayor número de destinatarios". ${ }^{3}$

Hasta acá pueden citarse investigaciones públicas sobre las consideraciones políticas de la distribución con justicia social del sistema de medio desde el rol que los Estados deben asumir para profundizar en la calidad de la democracia. Una calidad que inevitablemente debe ahondar en la tenencia de los medios de información, por ahora, a favor de las empresas mediáticas y no a la comunicación como un derecho humano.

Sin embargo, el interés de este artículo es aproximarse en la configuración de la estructura social resultante de esa tenencia favorable únicamente al mercado. ¿Cuáles son esos mapas de medios?, ¿cómo se distribuye ese sistema de medios, tanto desde las clases populares como desde las élites económicas y políticas de la región? Para hablar al respecto, se pueden evidenciar avances en la región en dos países.

\section{Los mapas de medios de Costa Rica y El Salvador}

Ambos países, al revisar su bibliografía, poseen estudios que convergen en puntos comunes. Por un lado, hay estudios para explicar los usos de la distribución del espectro radioeléctrico destinado a la radio y la televisión; y por otro, existen investigaciones sobre las configuraciones de élites económicas propietarias del sistema de medios privados y su paralelismo con el poder político derivadas de esa relación.

En el caso de Costa Rica, el estudio "El mundo de la radiodifusión en Costa Rica" apunta a que existe una "concentración geográfica" de la radiodifusión con "45 de 51" frecuencias, separadas a $400 \mathrm{Khz}$ privilegiando la transmisión desde la zona metropolitana de la capital, San José. Como consecuencia lógica, dejan descubiertas las zonas "rurales-costeras y fronterizas". Además, explican que existe una concentración de radios en pequeños grupos económicos. Los siguientes grupos radiofónicos acaparan juntos poco más del 50 por ciento del espectro radioeléctrico dedicado a la radiodifusión (Chinchilla 16). 
Tabla 2

PRINCIPALES GRUPOS MEDIÁTICOS POR NÚMERO DE FRECUENCIAS DE RADIO

\begin{tabular}{lc}
\hline Grupo & N. ${ }^{\circ}$ de frecuencias \\
\hline Cadena de emisoras Columbia & 12 \\
Grupo radiofónico Central & 11 \\
Cadena Radio Costarricense & 10 \\
Iglesia Católica & 10 \\
Comercializadora radial S. A. (vinculada al Grupo Extra) & 10 \\
\hline
\end{tabular}

Fuente: Elaboración propia a partir de Chinchilla (2013).

Chinchilla analiza cómo en la práctica se viola la institucionalidad de la normativa legal, al permitir el "arrendamiento", "traspasos", "prestamos", "subutilizadas" (fuera del aire) y "repetición de contenidos" (encadenadas), los cuales están debidamente regulados en la ley para evitar la concentración y optimizar un bien limitado y demenial (público). Nuevamente, el estudio se ve limitado ante la falta de información debido a que, según la Superintendencia de Telecomunicaciones (SUTEL), no posee información de cuántas emisoras existen en Costa Rica y plantea contradicciones con la Cámara Nacional de Radiodifusión (CANARA). Los datos, de acuerdo con diversas fuentes citadas, van desde 102 a 149 radioemisoras entre AM y FM; por lo consiguiente, debido a la falta de información y legalidad existen radios que operan sin permiso.

El debate de las radios sin permiso o en su forma peyorativa "radios piratas" beneficia a las mismas empresas privadas; sin embrago, acá emergen un conjunto de presiones, principalmente para las radios privadas con vocación de servicio comunitario, en buena medida, y es complejo para la pluralidad de sectores de la comunicación, porque, según se establece en el estudio, "aseguran no conocer ninguna experiencia de radios ciudadana o comunitaria". ${ }^{4}$

El caso de El Salvador es diferente. A partir de los años noventa se produjo un movimiento para la "democratización de la palabra", "en el mundo todos valemos, en el dial todos cabemos" son algunos eslóganes de la época que definían la búsqueda de la participación de la sociedad en el espectro radioeléctrico. El primer esfuerzo de configurar un mapa sobre la estructura de la radiodifusión salvadoreña surgió en 2011 y se enfocó en la banda FM. El mapa reveló que, de las 49 frecuencias FM, se han repartido 28 de transmisión nacional y 21 fragmentadas como radios locales y regionales (Pérez R. 185).

A diferencia de Costa Rica y el resto de países de la región, existe más claridad de cada uno de los propietarios de los medios y la concentración en grupos económicos 
derivados de esos oligopolios radiofónicos, clasificados en tres grupos del sector privado. El grupo primario está formado por el acaparamiento de frecuencias de transmisión nacional (cobertura total del territorio) y regionales. El grupo secundario está integrado por un grupo minoritario de radios de trasmisión nacional, pero sin participación local. El grupo terciario corresponde a los grupos mediáticos que concentran el mayor número de frecuencias regionales y locales entre las que se encuentran iglesias de vocación cristianas y católicas. El estudio resalta las radios nacionales católicas, evangélicas y pone de manifiesto la limitada participación de radios estatales. Entre sus resultados pone en perspectiva la presencia de las radios comunitarias aglutinadas dentro de una misma frecuencia 92.1 FM fraccionada en numerosas radios distribuidas a nivel local.

Tabla 3

DISTRIBUCIÓN PORCENTUAL POR GRUPO MEDIÁTICO EN RADIO FM

\begin{tabular}{lccccc}
\hline GRUPO & Grupos & Nacionales (A) & Locales (B) & $\%$ A & $\%$ B \\
\hline Primario & 6 & 16 & 38 & 57.14 & 20.87 \\
Secundario & 3 & 4 & 2 & 14.28 & 1.09 \\
Terciario & 15 & & 65 & & 35.71 \\
Católicas & & 2 & & 74.28 & \\
Evangélicas & 2 & & 7.14 & \\
Estatales & & & 63 & & 34.61 \\
Particulares & & & 14 & & 7.69 \\
Comunitarias & & & &
\end{tabular}

Fuente: Elaboración propia a partir de Pérez R. (2013).

\section{Relaciones de poder}

El énfasis de este estudio en las radios en propiedad de iglesias católicas y cristianas libres hace reflexionar en lo que se ha convertido desde la década de los setenta en un espacio importante para la interacción de redes de poder. Benedicte Bull se refiere a las iglesias evangélicas con una fuerte influencia con el "evangelio de la prosperidad" en donde convergen grupos de élites que operan en diferentes ámbitos culturales, política y negocios, desde donde interactúan en redes de poder y configuran alianzas con empresas internacionales que sirven para formar redes internacionales. Chomsky hace otra reflexión en virtud de la seguridad que brindaban las iglesias durante las 
dictaduras militares, y el compromiso social asumido durante la represión. Considera que a diferencia de espacios como sindicatos "las iglesias si existían de manera que las charlas y conferencias [políticas] se hacían con frecuencia en ellas: la solidaridad con Centroamérica se originó en su mayor parte en las iglesias" (16).

Las élites salvadoreñas han favorecido a la iglesia católica, entregándoles frecuencias de radio y televisión en comodato, superando los trámites de ley, para proyectos de rentabilidad económica pro obras sociales. Por el contrario, la izquierda salvadoreña encontró principalmente espacios a través de la "iglesia popular católica", y -también- la teología de la liberación del servicio jesuita.

Se puede afirmar que los medios vinculados a las iglesias católica y evangélica se han convertido en espacios de disputa política; primero por no ser medios que peleen el pastel publicitario altamente concentrado por los medios privados hegemónicos y han sido quienes mejor han limitado el acceso de los medios ciudadanos; segundo, porque también se han convertido en plataformas desde donde se puede hacer política partidaria. Guibson Dantas considera que la mediación a través de la fe puede constituir una instrumentalización de la sociedad, del pueblo, desde la hegemonía cultural que imponen las iglesias y además influenciar negativamente la libertad del ciudadano de tomar decisiones libres.

Para profundizar las relaciones de poder configurado en el sector privado de las comunicaciones en relación a su concentración horizontal (sector del infoentretenimiento); vertical (otros sectores de la economía) y sus vínculos con el poder político, existen dos estudios que abordan ambos fenómenos. En Costa Rica, el artículo "Los dueños de la palabra. Costa Rica en un contexto de reforma neoliberal" explora los procesos de transnacionalización de la economía en ese país, durante el nuevo modelo económico surgido en los años noventa y que "no ha excluido a las economías de los medios de comunicación costarricenses" (Robles y Voorend 158).

Presentan la forma en que directivos de grupos mediáticos han participado en puesto de dirección en un abanico de diversidad de rubros empresariales y, asimismo, en cargos públicos, en una especie de "paralelismo político" (Hallin y Mancini). El repunte económico de los empresarios de medios en Costa Rica ha estado influenciado por un proceso de desregulación desde 1994, cuando la Sala de lo Constitucional “elimina el Artículo 2 de la Ley 6220, encargada de regular los medios de difusión y en el cual se señalaba la propiedad nacional de los medios de difusión y agencias de publicidad" (Robles y Voorend 158).

Como cita el artículo, se han presentado dos procesos de transnacionalización de grupos mediáticos. Por un lado, la asociación del Grupo Nación con el grupo español PRISA y Caracol de Colombia. Por otro lado, la adquisición de grupos de radio y canales de televisión del mexicano, nacionalizado guatemalteco, Ángel González, 
quien controla el grupo televisivo REPRETEL y Central de Radios, solo compitiendo con TELETICA, del empresario costarricense Picado Cozza.

Tabla 4

CONCENTRACIÓN DE LOS GRUPOS MEDIÁTICOS DE TELEVISIÓN EN COSTA RICA

\begin{tabular}{ccc}
\hline Propietario & Grupo & Televisión \\
\hline Ángel González & REPRETEL & $2,4,6,9,11$ \\
Picado Cozza & TELETICA & 7,33 \\
& & Cable Tica \\
& & Cable Color \\
\hline
\end{tabular}

Fuente: Elaboración propia a partir de Robles y Voorend (2012).

Esta realidad en Costa Rica pone en alerta una diferencia central en la economía de los medios de comunicación menos internacionalizados. En el caso de El Salvador, según la ley de Telecomunicaciones de 1997, las élites del sistema de medios crearon un candado para mantener a salvo sus intereses nacionales en un gobierno que liberalizó todo, menos la radio y la televisión. El artículo $123^{5}$ de la citada ley reconoce que las concesiones de frecuencias solo pueden ser otorgadas a personas naturales por nacimiento o jurídicas salvadoreñas, y, en dado caso, su capital social será constituido el 50 por ciento más uno de salvadoreños.

De esta forma, la concentración quedó, a diferencia del resto de países de Centroamérica, en manos nacionales, lo cual configuró grupos mediáticos cuyas raíces se encuentran en los años cincuenta con el nacimiento de tres radios: Circuito YSR, YSU y la YSEB. Los socios de esas radios se convertirían posteriormente en propietarios de canal 2, 4, 6. Su figura más sobresaliente, Boris Eserski, es el fundador de Telecorporación Salvadoreña (TCS), el principal grupo mediático desde donde se ha ramificado toda una red de relaciones de amistades y exempleados entre los diferentes grupos de radio y televisión. Sintetiza estas relaciones de élites mediáticas en el artículo "El mapa de medios en El Salvador. Todo queda en familia” (Pérez R y Carballo W 47).

Este estudio no solo elabora un mapa de la adjudicación de frecuencias de radio y televisión, sino también revela las juntas directivas para establecer las relaciones históricas que configuraron una alianza de elites vinculadas en el tiempo para aglutinarse en la Asociación Salvadoreña de Radiodifusoras de El Salvador (ASDER). De este festín mediático, la televisión es el plato fuerte con alto nivel de concentración. 
Tabla 5

MAPA DE LA CONCENTRACIÓN DE LA TELEVISIÓN EN EL SALVADOR

\begin{tabular}{lcc}
\hline Grupo & Canales & $\%$ \\
\hline Tcs $(2,4,6,31,35)$ & 5 & 52.2 \\
Frecuencias a nombre de edu tv, tv independiente y tv juventud ${ }^{6}$ & 7 & 8.7 \\
MEGAVISIÓN (15 (no es nacional), 19, 21) & 2 & 13.0 \\
Cable frecuencias (53, 55, 59) & 3 & 4.3 \\
RSM (Canal 12) & 2 & 4.3 \\
Universidad Católica (57) & 1 & 4.3 \\
Tecno visión (33) & 1 & 4.3 \\
Iglesia ELIM (27) & 1 & 4.3 \\
RSM (37) (CANAL 11) & 1 & 4.3 \\
AGAPE CANAL 8 & 1 & \\
\hline
\end{tabular}

Fuente: Elaboración propia a partir del mapa de medios en El Salvador. Todo queda en familia (Pérez R. y Carballo W. 64).

Tabla 6

MAPA TELEVISIÓN DE TRANSMISIÓN NACIONAL

\begin{tabular}{lcc}
\hline Grupo & $\begin{array}{c}\text { N. }{ }^{\circ} \text { de frecuencias } \\
\text { regionales }\end{array}$ & Fragmento \\
\hline Gentv Canal 297 & 1 & 3 \\
Pedro Emilio Silhy Miguel. Canal 61 & 1 & 3 \\
Máxima Aceleración. Canal 63 & 2 & 3 \\
Máxima Aceleración. Canal 65 & 2 & 3 \\
Master Comunicaciones S. A. de C.V. Canal 67 & 1 & 2 \\
\hline
\end{tabular}

Fuente: Elaboración propia a partir del mapa de medios en El Salvador. Todo queda en familia (Pérez R. y Carballo W. 64).

En general, El Salvador ha avanzado sustancialmente en esclarecer las relaciones de poder resultantes de la explotación privada del espectro radioeléctrico para Radio y Televisión desde su distribución territorial. En su punto relevante hacia la internacionalización de las economías del infoentretenimiento en Centroamérica, 
hasta el 2013, emerge el empresario Ángel González con la compra del Canal 11, Canal 12 y Radio Sonora para conformar el grupo mediático RSM, Red de Medios Salvadoreños.

\section{La configuración de las radios comunitarias: Caso de El Salvador}

Por el contrario, frente a la integración económica de la región, se puede observar que, pese a que se ha hablado acerca de la televisión, es la radio en toda Centroamérica en donde se insertan procesos de comunicación ciudadana dentro de la estructura social. El Salvador es un ejemplo para tomarlo como caso de estudio en este artículo, en donde se caracterizan cinco momentos históricos del surgimiento de las radios comunitarias en El Salvador.

Primero: Radios comunitarias resultado de repoblaciones durante el conflicto armado antes de la firma de los Acuerdos de Paz en 1992. Comunidades que en un primer momento adquirieron terrenos, con el respaldo de la cooperación internacional y Amnistía Internacional.

El trabajo de estas comunidades consistía en la búsqueda de consensos para acuerdos sobre las relaciones sociales establecidas en torno a una identidad común, luego de asentarse en los nuevos territorios. Veían la necesidad de comenzar por delimitar los terrenos, a fin de definir las respectivas zonas agrícolas, habitacionales y públicas. Fueron los fundadores de estas radios quienes vieron en la comunicación la posibilidad de utilizar el medio para fines colectivos y la construcción de sentido en relación a un proyecto histórico (Montoya). Después de más de 20 años, muchas de estas radios aun buscan modelos de producción alternativos; centran su atención en la memoria histórica como vínculo de su identidad. Las comunidades expresan un alto vínculo con las radios en cuanto a sentido de propiedad, unidos por la historia.

Segundo: Radios resultado de la adquisición de la frecuencia 92.1 FM. En 1995, las radios comunitarias fueron reprimidas por la Policía Nacional Civil con una orden judicial en contra del uso ilegal del espectro radioeléctrico. ${ }^{8}$ Para organizarse, fundan la Asociación de Radios y Programas Participativos (ARPAS), quienes, junto con la cooperación internacional, obtuvieron la donación para comprar, bajo las reglas del mercado, la frecuencia 92.1. El objetivo era legalizar las radios comunitarias existentes y ampliar nuevos proyectos locales. El resultado, según datos de la Superintendencia General de Electricidad y telecomunicaciones (SIGET) fue la expansión a 16 radios que cohabitan en la misma frecuencia.

Tercero: Radios que participaron y ganaron procesos de subasta, a partir de la nueva Ley de Telecomunicaciones. Históricamente puede observarse que la mayoría de radios comunitarias que participaron en el proceso de subasta perdieron, pese a encontrarse en zonas poco atractivas para el mercado. Si existe un subsector que se desprende del sector privado interesado en participar en grandes zonas de poblaciones son las iglesias cristianas. Sin embargo, se reportan casos en donde nadie demostró interés, 
ni la empresa privada, ni las iglesias acudieron al proceso de subasta, de manera que se obtuvo la frecuencia solo después de pagar la base de la oferta. Así se encuentran: Radio San Pedro, departamento de Cabañas, en la frontera con Honduras; La nueva radio Farabundo Martí, en Arcatao, Chalatenango, un municipio histórico por las masacres a pobladores durante el conflicto armado y, anteriormente, radio Izcanal, Asulután, obtuvo una frecuencia que alquila para lograr sostener su sistema de radio y televisión.

Cuarto: Durante el primer gobierno del FMLN 2009-2014, concesionaron frecuencias oficiales para ser administradas por alcaldías o proyectos comunitarios ya existentes. Esta modalidad ya había sido aplicada por anteriores gobiernos, pero fueron para beneficiar a la Iglesia católica.

Por último, se configura un quinto momento. A partir del reconocimiento de los medios sin fines de lucro en la reforma a la Ley de Telecomunicaciones, el art. $5^{9}$ institucionaliza los medios ciudadanos, comunitarios, e incorpora a la misma enmienda las iglesias. Esta nueva normativa, excluida del proceso de subasta, posibilitará nuevos medios del sector.

\section{La configuración del mapa de medios por indicadores de actividad económica}

Para construir políticas públicas de comunicación se requiere de producir datos o indicadores que permitan observar cómo se puede potenciar el pluralismo mediático desde los sectores y los territorios. Hasta este momento los estudios en El Salvador demuestran una distribución amorfa, desde el punto de vista de acceso, que obedece, principalmente, a las lógicas del mercado.

Para elaborar la relación del sistema de medios de información en caso de las radios FM, se tomó como referencia el estudio de Salvador Arias (Atlas de la pobreza y la opulencia en El Salvador), donde el autor elabora un mapa sobre la condición de los 262 municipios del territorio salvadoreño, con relación a su actividad económica.

De acuerdo con los datos recogidos, determinó que existen 100 municipios más pobres, en donde más del 50 por ciento de la población vive en miseria, porque tienen ingresos inferiores a la canasta básica alimentaria. ${ }^{10}$

Arias cruzó la información entre el informe del PNUD, FLACSO, MINED "Mapa de pobreza y exclusión social" y el VII Censos económicos, por estar disponible en el momento del estudio. A partir de los datos se clasifican los municipios de un departamento de acuerdo con la posición económica que ocupa según el número de establecimientos que generan empleo.

El censo económico demuestra que en El Salvador existen 22780 empresas en la gran industria, quienes contratan más de 5 trabajadores. En total estas empresas emplearon 164580 personas remuneradas, que devengaron USD 781 899.420. Los 31070 propietarios y socios de estas empresas generaron un valor agregado censal 
de USD 2007371.742 en el año del censo. Entre estos empresarios se encuentran un limitado grupo de 41 principales empresas mediáticas concentradas en El Salvador, capaces de configurar la agenda informativa y cultural.

A partir de esta información, en el 2015, se elaboró un cuadro que expresa cómo el sistema de medios privado se reproduce en los territorios de mayor actividad económica y, por el contrario, los medios del tercer sector "comunitarios" participan en los municipios de menor actividad. Queda claro que la explotación que realiza el medio depende de la forma en que instrumentalizan o "mercantilizan las audiencias", a través de la "propensión al consumo"; a cambio de un servicio público mediado, principalmente, por la búsqueda de la rentabilidad económica.

En la Tabla 6 aparece la posición en que se encuentra el municipio en relación a la actividad económica, considerando que solo en 58 de 262 municipios existen proyectos radiofónicos. Esto es el 21.96 por ciento de la administración territorial de El Salvador (Pérez 188).

Los resultados del mapeo territorial demuestran la tendencia del modelo de negocios de las radios en participar en ciudades con mayor capacidad de consumo; por el contrario, los proyectos comunitarios tienden a participar en ciudades con menor actividad económica. De hecho, existen casos de radios comunitarias con esfuerzos en ciudad cabecera que han cerrado debido a la presión económica de subsistencia y mayor grado de competencia, entre otras variables. El fenómeno de las radios comunitarias tiende a ser más evidente en el oriente del país. 
Tabla 7

CUADRO COMPARATIVO DE PRESENCIA DE RADIOS POR ACTIVIDAD ECONÓMICA

\begin{tabular}{|c|c|c|c|c|c|}
\hline Departamento & $\begin{array}{c}\mathrm{N} .^{\circ} \\
\text { municipios }\end{array}$ & & $\begin{array}{c}\text { Posición por actividad } \\
\text { económica }\end{array}$ & $\begin{array}{l}\mathrm{N} .{ }^{\circ} \text { radios } \\
\text { comerciales }\end{array}$ & $\begin{array}{c}\text { Radios } \\
\text { comunitarias }\end{array}$ \\
\hline \multirow{3}{*}{ Santa Ana } & \multirow{3}{*}{13} & 1 & Santa Ana & \multirow{4}{*}{18} & \multirow{4}{*}{3} \\
\hline & & 2 & Chalchuapa* & & \\
\hline & & 3 & Matapán* & & \\
\hline \multirow{6}{*}{ Ahuachapán } & \multirow{6}{*}{12} & 6 & Coatepeque* & & \\
\hline & & 1 & Ahuachapán* & \multirow{5}{*}{11} & \multirow{4}{*}{3} \\
\hline & & 2 & San Francisco Menéndez & & \\
\hline & & 3 & Texistepeque & & \\
\hline & & 5 & Jujutla* & & \\
\hline & & 1 & Sonsonate* & & \multirow{5}{*}{2} \\
\hline \multirow{3}{*}{ Sonsonate } & \multirow{3}{*}{16} & 2 & Acajutla* & \multirow{4}{*}{16} & \\
\hline & & 3 & Izalco & & \\
\hline & & 4 & Armenia & & \\
\hline \multirow{7}{*}{ La Libertad } & \multirow{7}{*}{22} & 5 & Juayua & & \\
\hline & & 6 & La Libertad & \multirow{6}{*}{5} & \multirow{6}{*}{2} \\
\hline & & 8 & Zaragoza* & & \\
\hline & & 10 & Jayaque & & \\
\hline & & 13 & Nuevo Cuscatlán** & & \\
\hline & & 14 & Teotepeque & & \\
\hline & & 1 & San Salvador* & & \\
\hline \multirow{2}{*}{ San Salvador } & \multirow{2}{*}{19} & 13 & Nejapa* & \multirow{2}{*}{51} & \multirow[t]{2}{*}{3} \\
\hline & & 16 & Guazapa* & & \\
\hline \multirow{5}{*}{ Chalatenango } & \multirow{5}{*}{33} & 1 & Chalatenango & \multirow{5}{*}{7} & \multirow{5}{*}{3} \\
\hline & & 2 & Nueva Concepción ** & & \\
\hline & & 5 & San Ignacio & & \\
\hline & & 32 & San Isidro Labrador* & & \\
\hline & & 31 & Arcatao* & & \\
\hline \multirow{2}{*}{ Cuscatlán } & \multirow{2}{*}{16} & 1 & Cojutepeque & \multirow{2}{*}{4} & \multirow{2}{*}{1} \\
\hline & & 2 & Suchitoto* & & \\
\hline La Paz & 22 & 1 & Zacatecoluca & 0 & 0 \\
\hline
\end{tabular}




\begin{tabular}{|c|c|c|c|c|c|}
\hline Departamento & $\begin{array}{c}\mathrm{N} \cdot{ }^{\circ} \\
\text { municipios }\end{array}$ & & $\begin{array}{c}\text { Posición por actividad } \\
\text { económica }\end{array}$ & $\begin{array}{l}\mathrm{N} .{ }^{\circ} \text { radios } \\
\text { comerciales }\end{array}$ & $\begin{array}{c}\text { Radios } \\
\text { comunitarias }\end{array}$ \\
\hline \multirow{3}{*}{ Cabañas } & \multirow{3}{*}{9} & 1 & Sensuntepeque & \multirow{3}{*}{4} & \multirow{3}{*}{2} \\
\hline & & 2 & Ilobasco & & \\
\hline & & 6 & Victoria* & & \\
\hline \multirow{2}{*}{ San Vicente } & \multirow{2}{*}{13} & 1 & San Vicente & \multirow{2}{*}{5} & \multirow{2}{*}{1} \\
\hline & & 3 & Tecoluca* & & \\
\hline \multirow{3}{*}{ San Miguel } & \multirow{3}{*}{20} & 1 & San Miguel & \multirow{3}{*}{23} & \multirow{3}{*}{1} \\
\hline & & 4 & Ciudad Barrios* & & \\
\hline & & 5 & San Jorge & & \\
\hline \multirow{8}{*}{ Usulután } & \multirow{8}{*}{23} & 1 & Usulután & \multirow{8}{*}{24} & \multirow{8}{*}{2} \\
\hline & & 3 & Santiago de María & & \\
\hline & & 4 & Jucuapa & & \\
\hline & & 5 & Berlín & & \\
\hline & & 7 & Santa Elena & & \\
\hline & & 18 & Alegría & & \\
\hline & & 20 & Nueva Granada * & & \\
\hline & & 17 & Jiquilisco* & & \\
\hline \multirow{5}{*}{ Morazán } & \multirow{5}{*}{26} & 1 & San Francisco Gotera & \multirow{5}{*}{14} & \multirow{5}{*}{1} \\
\hline & & 3 & Corinto & & \\
\hline & & 6 & Cacaopera & & \\
\hline & & 7 & Meanguera* & & \\
\hline & & 8 & Chilanga & & \\
\hline \multirow{4}{*}{ La Unión } & \multirow{4}{*}{18} & 1 & La Unión & \multirow{4}{*}{14} & \multirow{4}{*}{1} \\
\hline & & 2 & Santa Rosa de Lima & & \\
\hline & & 3 & Conchagua * & & \\
\hline & & 4 & Pasaquina & & \\
\hline
\end{tabular}

Fuente: Elaboración propia a partir de VII Censos económicos 2005 y Asociación de Radios y Programas participativos, ARPAS. *Municipio con radio comunitaria. ${ }^{* *}$ Municipio de radios oficiales de las alcaldías.

El panorama de la televisión nacional tampoco es alentador. La concentración de medios se prolifera desde las 3 ciudades cabeceras departamentales más importantes 
de El Salvador. A nivel de estudios sobre el índice de concentración de audiencia de la televisión se tiene información sobre su comportamiento. El Estado salvadoreño, a través de la Superintendencia de Competencia, SC, encargó una investigación del nivel de concentración de mercado y audiencia en la televisión. De acuerdo a los datos arrojados Telecorporación Salvadoreña (TCS) tiene un índice de IHH (Herfindahl o Índice de Herfindahl e Hirschman) que mide la concentración de mercado mostrando un valor 2.550, límite entre moderada y alta; sin embargo, el índice de dominancia, ID, marca una concentración alta con 3906. El uso de la banda VHF y UHF, índice C2 alcanza el 62.50 por ciento (Greco 83).

Tabla 8

PARTICIPACIÓN DE MERCADO SEGÚN RATING POR GRUPO ECONÓMICO, ÍNDICES DE CONCENTRACIÓN DOMINANCIA Y RIVALIDAD

\begin{tabular}{lrr}
\hline Grupo propietario & \multicolumn{1}{c}{2013} & \multicolumn{1}{c}{2014} \\
\hline TCS (canales 2, 4, 6 y 35) & $69.02 \%$ & $65.99 \%$ \\
Megavisión (canales 15, 19 y 21) & $21.40 \%$ & $21.37 \%$ \\
Canal 12 & $4.99 \%$ & $5.93 \%$ \\
Tecnovisión (Canal 33) & $2.44 \%$ & $1.96 \%$ \\
TV Oriental (Canal 23) & $2.14 \%$ & $4.74 \%$ \\
\hline Total & $100.00 \%$ & $100.00 \%$ \\
& & \\
C2 & $90.42 \%$ & $87.37 \%$ \\
Índice de concentración IHH & 5.258 & 4.873 \\
Índice de dominancia & 8.287 & 8.075 \\
Grado de rivalidad & & 0.04 \\
\hline
\end{tabular}

Fuente: Informe, Condiciones de competencia en la televisión abierta en El Salvador (Greco 83).

En sus conclusiones confirma que:

Actualmente el Grupo [TCS] cuenta con una participación medida en rating de audiencia del $66 \%$ según datos de 2014 y del 71 \% en términos de su participación en la inversión publicitaria en TV abierta (que ha sido estimada tomando como proxy los ingresos de los canales de TV abierta, según sus estados financieros 2013).

Sus más cercanos competidores: el grupo Megavisión, y Canal 12 (RSM), del empresario Ángel González aún no representa una competencia significativa "cuya 
participación en el mercado es menor, tanto en términos de rating de audiencia como en términos de participación en inversión publicitaria en TV abierta" (Greco 108).

En buena medida, obedece a que desde la década de los sesenta cuando se conformó $\mathrm{TCS}^{11}$ no ha existido transparencia en la adjudicación de las frecuencias, revelado por un informe de la Secretaria de Transparencia de la Presidencia en donde explican que el "60\%" de las frecuencias del espectro radioeléctrico para radio y televisión no poseen documentación "que registre la entrega de la concesión, autorización, licencias, convenios, acuerdos o permisos" (SPTA 29). El caso del grupo TCS en su conformación histórica mantiene "unas ventajas sustancialmente nunca desafiadas de primer jugador".

El mapa de medios de El Salvador no presentará cambios significativos en el futuro. Los recursos de inconstitucionalidad 65-2012, presentado por el movimiento ciudadano, relacionados a la renovación automática de frecuencias fueron resueltos a favor de la estructura de medios existente. Es decir, por los próximos 20 años a partir del 2017, ni los grupos mediáticos internacionalizados, ni al extremo de la estructura, las radios comunitarias presentarán cambios sustanciales. La Sala de lo Constitucional, de la Corte Suprema de Justicia de El Salvador priorizó y valoró la seguridad jurídica de los derechos económicos, y esto les beneficiará en el proceso de la digitalización a partir del 2017.

Las concesiones para los servicios de radiodifusión sonora y de televisión de libre recepción que pudieran haber sido otorgadas previo a la emisión de este fallo, deberán mantenerse conforme a la legislación vigente antes del presente fallo, es decir, conservará el beneficio de la prórroga del plazo concedido por la ley, previa verificación por la SIGET (Inc. 65-2012/36-2014, 2015, 58).

Estos estudios del sistema de medios realizados por el Estado han contribuido a transparentar las condiciones de las empresas mediáticas, tendientes a reproducir las condiciones de desigualdad entre los diferentes sectores de la comunicación, desde los sectores, como desde los territorios, como ya se ha explicado anteriormente. Y es la televisión quien mantiene los índices más altos de concentración, desde las audiencias, el mercado y desde la centralización en las ciudades de mayor actividad económica.

\section{El caso de Ángel González}

Como se ha explicado al principio de este artículo, la económica de la región comienza a reconfigurarse alrededor de una nueva élite económica de la región vinculada al sector de la infocomunicación. En El Salvador, el empresario Ángel González representa una reagrupación del sistema de medios nacionales, en donde se ha sorteado las normativas nacionales a partir del 2013 para configurar un nuevo grupo mediático en disputa del poder económico, representado en la hegemonía de TCS. 
En conjunto se ha abierto un ámbito de pluralidad en el sector económico privado para configurar un grupo contrahegemónico internacional.

El panorama que se abre con Ángel González en El Salvador refleja la flexibilidad ideológica en que se mueve en toda la región. Por ejemplo, en Nicaragua, el observatorio de medios CINCO valora un cambio de correlación mediática con la llegada del gobierno de Daniel Ortega, quien, en alianza con el citado Ángel González, desde 2010 ha incursionado en la televisión y la radio. El informe lo considera un duopolio televisivo (Rothschuh).

En El Salvador, Ángel González, apareció en diciembre de 2011 con la concesión de la radio YSCE 680 AM por un valor de USD 62 500, muy por encima del precio logrado en otras subastas de frecuencias AM, según reporta la SIGET. A partir de la fecha, una serie de cambios en la Superintendencia se fueron sucediendo, al cierre del gobierno de Mauricio Funes, primer presidente del gobierno del FMLN. Primero el nombramiento del superintendente en 2014, Astor Escalante, quien en sus primeras acciones sometió a concurso 6 frecuencias de televisión, presuntamente para ser adquiridas por una empresa relacionada con González, en una alianza política con Funes y una fracción del FMLN. El proceso fue suspendido, cuando, por primera vez en la historia, los intereses de la gremial de medios privados ASDER y el Movimiento por la Democratización de la Comunicación se unieron en contra de la amenaza del capital internacional. Posteriormente, la compra de canal 37 es trasladada preferentemente a Canal 11; y, por último, la compra de Canal $12 .{ }^{12}$

Las relaciones de Ángel González han sido la tónica en gobiernos de izquierda y derecha, desde Suramérica a Centroamérica. De ahí, Guatemala, Honduras y Costa Ricas son claros ejemplos de su alto nivel de influencia mediática de transmisión nacional, en alianzas estratégicas con el poder político y económico. Basta ver el mapa de medios para constatar su influencia, según datos revelados en su sitio (web http:// www.albavision.tv), posicionando sus medios en las bandas VHF y FM entre los de mayor índice de recepción, ejemplificados en el caso Costa Rica y de transmisión nacional en El Salvador.

Tabla 9

CUADRO DE MEDIO DEL GRUPO ALBAVISIÓN (ÁNGEL GONZÁLEZ)

\begin{tabular}{lccccc}
\hline País & Guatemala & El Salvador & Honduras & Nicaragua & Costa Rica \\
\hline Televisión & 5 & 2 & 6 & 7 & 4 \\
Radio & 13 & 1 & 1 & 12 & 11 \\
\hline
\end{tabular}

Fuente: Elaboración propia. 


\section{El enfoque estructural de la cultura}

Luego de estas valoraciones de la estructura social determinada por las interacciones sociales mediadas por lo económico, no se puede dejar de lado y se dedicará unas líneas a comprender la ampliación hacia la estructura cultural que se desprende en los territorios. Thompson, J. hace referencia al espacio temporal en donde se "pone de relieve la preocupación por los contextos y procesos estructurados socialmente donde se insertan las formas simbólicas" (204). Considera estas formas simbólicas bajo 5 aspectos: intencional, convencional, estructural, referencial y contextual.

De manera particular se explica el aspecto contextual como parte del proceso socio histórico, en donde se producen y reciben las formas simbólicas estructuradas socialmente. Por lo tanto, estas formas simbólicas son constantemente valoradas o interpretadas entre los sujetos que interactúan en contextos espacio temporales específicos, siguiendo ciertas trayectorias determinadas por el volumen y la distribución de recursos y capital: capital económico, capital cultural y capital simbólico. Este último incluye "los elogios, el prestigio y el reconocimiento acumulados que se asocian a una persona o una posición" (220).

Los campos de interacción, en donde interactúan los sujetos, se organizan en instituciones a través de reglas y recursos integradas a relaciones sociales establecidas por ellas. Estas dan forma a campos de interacción preexistentes, pero, sobre todo, crean "un nuevo conjunto de posiciones y trayectorias posibles" (223). Las estructuras sociales, por tanto, establecen los campos de interacción y las instituciones a partir de relaciones de poder asimétricas, estables y perdurables. Define el poder como la capacidad del individuo de actuar y "alcanzar los objetivos e intereses que se persiguen" y "depende de la posición que se ocupa en un campo o en una institución", lo cual lo posibilita de cambiar o seguir los trayectos que desee. Es así como el poder dentro de los campos y las instituciones crean, entre los sujetos, relaciones de dominación y subordinación a partir de la capacidad parcial que tengan de los recursos. Si bien esto conduce al análisis de relación capital/salario, Thompson considera que también las formas de dominación son a través de la producción y la recepción de las formas simbólicas o contenidos simbólicos, dotándoles de sentido activo y creador.

Las formas simbólicas son recibidas por individuos que se sitúan en contextos socio históricos específicos, y las características sociales de esos contextos moldean las maneras en que son recibidas, comprendidas y valoradas por ellos (Thompson 227).

En las sociedades capitalistas modernas, la producción de contenidos simbólicos ha sido determinada por una valoración económica que constituye una mediación del consumo de estos. Eso mismo es lo que el actual sistema de medio reproduce como cultura dominante. Por el contrario, cuando se reconoce y se institucionaliza el sistema 
de medios ciudadanos se abre un nuevo campo de interacción que posibilita nuevos y variados flujos de formas simbólicas.

El sistema de medios ciudadanos se inserta en lo local y se separa de las grandes ciudades con alto capital económico en donde se homogeniza el consumo simbólico. Al mismo tiempo no se puede obviar el debate de lo tradicional frente a la cultura modernizante para construir interacciones con alto "capital cultural y capital simbólico". Es decir, las posibilidades de rescate, preservación de conocimientos, educación y construcción de identidad y prestigio. Ya sea desde la producción o la reproducción entre los miembros de la comunidad de comunicadores se configuran fortalezas en el intercambio de las formas simbólicas.

La posibilidad de comunicación de nuevos actores comunitarios, más cercanos y las posibilidades de crear nexos comunales constituyen fuentes de estudio que aún no se han investigado en la región para comprender los aportes que un sistema de medios culturalmente plural y democrático demandan para potenciar otra estructura con perspectiva de transformación social. Si bien es cierto que este tema puede ampliarse en otra publicación, se retoma para hacer notar que es necesario integrar la relación entre la economía y la cultura. El enfoque de este artículo ha centrado su atención en las industrias culturales más mediáticas, ahora se tratará la rentabilidad social que configura o reafirma otra estructura social posible.

\section{Red Centroamericana de Radios Comunitarias Indígenas}

Un caso reciente es la integración regional de medios comunitarios que nace desde los espacios destinados a la identidad y la cultura. La Red Centroamericana de Radios Comunitarias Indígenas aglutina esfuerzos de proyectos radiofónicos de los países: Guatemala, Belice, El Salvador, Honduras, Nicaragua, Costa Rica y Panamá. Desde este nuevo campo de interacción pueden institucionalizarse y unificar voluntades e intereses en la región global a través de una agenda pluricultural u otra cultura alterna a la dominante. La misión de la red establece que:

Somos un movimiento de radios comunitarias indigenas que desarrolla procesos de incidencia política, fortalece la identidad de los pueblos y saberes ancestrales a través de la capacitación, la democratización de la comunicación, la pluralidad de pensamiento, la equidad de género y la libre expresión.

El esfuerzo de esta red responde a la lógica de que mientras las economías regionales se globalizan, los problemas sociales lo hacen de la misma manera, de ahí la necesidad de globalizar los movimientos. En términos de Zallo esta perspectiva se delimita al considerar la diferencia entre "globalización capitalista" de "localismo, globalizado", en donde "unos lugares tienen un peso decisivo en el mundo global y unos son solo partes de una gran red" (Zallo 51). 
La experiencia de esta red es reciente, pero pone en contraste la articulación de movimientos sociales globalizados unificando los esfuerzos con la democratización de la comunicación. Su accionar se describe en las posibilidades de visibilizar un sector que en su praxis puede reivindicar los contenidos simbólicos de su cosmovisión, en campos de interacción globales, formas simbólicas en resistencia mesoamericana. Uno de sus desafíos constituye "replantearnos observar cómo la cosmovisión de nuestros pueblos resignifica el lenguaje y los formatos radiofónicos modernos", según lo establece la memoria de la reunión estratégica en junio de 2016. Tal como en su momento lo planteó Ramiro Beltrán, quien en sus últimos trabajos enfatizó en la "historia de la comunicación pre y poscolonial" para desmitificar la visión eurocéntrica de la comunicación. Significa la posibilidad de una producción centrada en la cantidad de contenido simbólico que posibilita una comunicación tecnológica más variada que reconfigura poderes en una estructura social que emerge desde la conciencia indoamericana.

Este apartado hace una reflexión de tipo ensayista, para estudiar las posibilidades de estas radios en campos de sobrevivencia de formas de interacción social que riñen con la modernidad anclada en la resistencia que les permite las raíces tradicionales. Quizá se trata de una descolonización de lo que hoy llamamos comunicación que conduce a encontrarse con una identidad y pensamiento latinoamericano. Quijano en "Colonialidad del poder, eurocentrismo y América Latina" recuerda que el encubrimiento de los pueblos originarios significó desde la colonia una "división racial del trabajo" que organizó una nueva estructura global y de control del trabajo de los recursos y los productos.

Todas las experiencias, historias, recursos y productos culturales, terminaron también articulados en un solo orden cultural global en torno de la hegemonía europea u occidental. En otros términos, como parte del nuevo patrón de poder mundial (Quijano 209).

La nueva praxis de los medios ciudadanos indígenas es una posibilidad para comprender las narrativas, el pensamiento, los símbolos, la organización; nuevos y variados contextos sociales desde donde se insertan las formas simbólicas. Es una oportunidad y una posibilidad para la cosmovisión mesoamericana en Centroamérica global en contraste, con la internacionalización de las economías. Sin caer en el romanticismo, la tarea está enunciada, aun cuando queda mucho por avanzar.

Para comprender las condiciones en que se encuentran estos esfuerzos, se presenta un balance en la región que evidencia las luchas sociales de las radios indígenas: 
Guatemala: La conformación del movimiento de radios comunitarias con una tradición fuerte de radios indígenas, no logró conquistar su lucha en el parlamento con la iniciativa 4087 para medios comunitarios.

Honduras: El decreto presidencial que reservaba el 31 por ciento para radios comunitarias se diluyó al incorporar a radios religiosas. Aun así, la Asociación de Medios Comunitarios de Honduras, $\mathrm{AMCH}$, coordina una mesa de radios comunitarias indígenas.

Nicaragua: Las relaciones del Estado con los pueblos indígenas del Caribe sur abren las posibilidades de concesionarles frecuencias; sin embargo, el panorama de un país con pocos recursos económicos no contribuye a una "comunicación integral", consideran los activistas de Nicaragua.

Costa Rica: Como se planteó anteriormente, no se reconoce ninguna radio comunitaria, pero entre 15 radios culturales existen tres ubicadas en pueblos indígenas, en un contexto de poco ordenamiento de la distribución del espectro.

El Salvador: Un país con heridas profundas del etnocidio indígena del 1932, sostuvo una radio indígena en la zona de la masacre, en la ciudad de Izalco; sin embargo, desapareció y ahora encabezan un nuevo esfuerzo para fundar otro proyecto de radio indígena. ${ }^{13}$

\section{Palabras finales}

La región centroamericana tiene mucho camino por avanzar en la transparencia del uso del espectro radioeléctrico y la configuración de sus relaciones de poder asimétrico. El Movimiento Social para la Democratización de la Comunicación ha dado pasos en el camino de investigar el fenómeno; sin embargo, no hay suficiente información para una prospectiva de políticas públicas en los países de la región centroamericana para superar las consecuencias sociales, resultado de la estructura de medios.

El Salvador parece ser el único país que mantiene un interés de profundizar en la discusión pública de la concentración del sistema de medios, integrándose la academia y las instituciones estatales, administradas por el gobierno del FMLN. Puede observarse que, junto a la presión del movimiento ciudadano, lograron las reformas de la Ley de Telecomunicaciones del 2016 e institucionalizar el tercer sector de la comunicación. Para el resto de países, el debate aún sigue vigente. 
El contexto en Nicaragua, bajo el gobierno del Frente Sandinista de Liberación Nacional, FSLN, el panorama es diferente. El informe "Estado de la Libertad de Expresión (2010-2013)" considera que "determinar el número real de radioemisoras existentes en el país, cada vez más resulta imposible. Ni siquiera para el sector académico esta información está disponible. La falta de transparencia es absoluta" (Rothschuh 9).

La presencia de Ángel Gonzálezy su pragmatismo en la región no es de extrañar. Tal como se inició este artículo, Centroamérica y su sistema de medios se están globalizando en alianzas políticas. En el caso salvadoreño, esta apertura va cediendo a nuevos concesionarios e indica que la pluralidad de medios obedece más a la lógica de los intereses de mercado del sector privado.

Aunque aún es temprano valorar la primera normativa en Centroamérica que legaliza la coexistencia del tercer sector en El Salvador, solo el tiempo dirá si las políticas públicas de comunicación caminan hacia empoderar a la ciudadanía o si las relaciones de poder con alianzas económicas del capital global mantendrán la dominación de una estructura social desigual y poco democrática. Tal como lo diría Ramiro Beltrán, después de la experiencia de 40 años por democratizar las comunicaciones en Iberoamérica, es necesario la "reinvención de la utopía por medio de nuevas políticas" a través de un "programa regional cooperativo".

En la región se hace necesario que la investigación académica se active en estos temas, en el estudio del poder económico y cultural. Es necesario Integrar el enfoque económico con enfoques estructurales de la cultura que posibilite estudiar la diversidad en la producción y el consumo de bienes del capital cultural y simbólico entre la comunicación ciudadana dentro de la reconfiguración de una nueva estructura social. El debate social de estos temas debe llevar a los estados a crear políticas públicas de comunicación.

\section{Notas}

1 Grupos de élites individuales que, debido a su control sobre natural, económico, político, social, organizacional o de coerción se colocan en una posición privilegiada para formalmente y o informalmente influir en las decisiones y prácticas (Bull).

2 Alex Segovia utiliza el término derecho de picaporte, como el derecho que se adjudican un club de miembros de las esferas de poder económico para de tocar la puerta y comunicarse informalmente con las más altas autoridades de los países en cualquier momento y circunstancia, una forma de interacción social que baña y aglutina los tres poderes del Estado.

$3 \mathrm{CIDH}$, La Colegiación Obligatoria de Periodistas (arts. 13 y 29 Convención Americana sobre Derechos Humanos). Opinión Consultiva OC-5/85 del 13 de noviembre de 1985. Serie A N. 5, párr. 31.

En Costa Rica las comunidades presentan múltiples necesidades de comunicación, pero como nunca ha habido medios comunitarios, simplemente no se los imaginan. No creen posible que puedan existir... Aquí la radio es para escuchar música y para que los comerciantes se anuncien; no se concibe que la radio pueda ser un instrumento de comunicación y expresión 
de los pueblos, ni como elemento fundamental en los procesos de construcción política de las organizaciones y las comunidades" (Chinchilla 22).

Art. 123. Las concesiones y licencias para los servicios de Difusión de Libre Recepción solo se otorgarán a personas naturales por nacimiento o jurídicas salvadoreñas. En el caso de personas jurídicas salvadoreñas, el capital social deberá ser constituido por lo menos con el cincuenta y uno por ciento de salvadoreños. Este capital social y sus reformas deberán ser reportados a la SIGET (Ley de Telecomunicaciones).

6 Un informe de transparencia advierte que estas frecuencias han sido adjudicadas de manera poco transparente y el mismo estudio de este cuadro explica la forma en que socios de TCS utilizando prestanombres usufructúan estas frecuencias sin transmisión de contenidos. Según información no oficial, aseguran que estas frecuencias fueron devueltas al Estado en enero de 2017.

7 Esta frecuencia fue adquirida en 2013 por el partido Frente Farabundo Martí, FMLN, para fundar canal Gentv, propiedad del partido de gobierno FMLN.

Periódico La Prensa Gráfica del 4 de diciembre de 1994.

Art. 5 “MEDIOS COMUNITARIOS Y OTROS OPERADORES SIN FINES DE LUCRO”.

Para 2007, la Canasta básica alimentaria fue de USD 156. 38; la canasta de sobrevivencia de USD 352.77; la canasta de mercado de USD 721.37. Encuesta de Hogares y Propósitos Múltiples, EHPM, 2007.

11 Para mayor información ver el mapa de medios en El Salvador "Todo queda en Familia".

12 Ver nota Romero, F. Séptimo Sentido “Las frecuencias de Ángel González en El Salvador". La Prensa Gráfica, 7(307). 2014. Recuperado de https://www.laprensagrafica.com/revistas/Lasfrecuencias-de-Angel-Gonzalez-en-El-Salvador-20140601-0093.html

13 Los datos acá presentados corresponden a la memoria de Reunión de Planificación Estratégica del Consejo Regional de la Red Centroamericana de Radios Comunitarias Indígenas La Antigua, Guatemala, junio 8 y 9, 2016.

\section{Bibliografía}

Arias, Salvador. Atlas de la pobreza y la opulencia en El Salvador. San Salvador, El Salvador, 2010. ALER/AMARC. Democratizando la palabra: Informe regional sobre marcos regulatorios de la radiodifusión en Centroamérica. El Salvador, 2009.

Benedicte, B. Governance in the Aftermath of NeoLiberalism: Aid, Elites and State Capacity in Central America. Forum for Development Studies, 2016. Recuperado de http://dx.doi.org/10.1 080/08039410.2015.1134647

Ramiro Beltrán, Luis. “Memoria y retos de futuro". Revista TELOS, 72(2007): 20-30.

Ramiro Beltrán, Luis. La Comunicación antes de Colón. Bolivia: CIBED, 2008.

Chinchilla, L. "El mundo de la radiodifusión en Costa Rica. Red de Medios e iniciativas de comunicación alternativa". RED. 2013.

Chomsky, Noam y Ramonet, Ignacio. Cómo nos venden la moto: información, poder y concentración de medios. Barcelona, España: Icarea editorial, S. A., 2002. 
Greco. E. Informe: Condiciones de competencia en la televisión abierta en El Salvador en línea. El Salvador: Superintendencia de Competencia. Recuperado de http://sc.gob.sv/ uploads/est_23_inf.pdf

Guibson Dantas, José. Neopentecostais e as mediações culturais. Recife, Brasil: Livro RápidoElógica, 2007.

Hallin, Daniel y Mancini Paolo. Sistemas mediáticos comparados. Tres modelos de relación entre los medios de comunicación y la política. Barcelona, España: Hacer, 2008.

Mastrini, G. y Becerra, M. (Ed.). Los Monopolios de la verdad: descifrando la estructura y concentración de los medios en Centroamérica y República Dominicana. Buenos Aires, Argentina: Prometeo Libros, 2009.

Montoya, Aquiles. “¿Es posible abatir la pobreza en el capitalismo?” Revista ECA 5 (1998): 91-92. 1998.

Moreno, Raúl. La globalización neoliberal en El Salvador: Un análisis de sus impactos e implicaciones. Barcelona, España: Fundación Mon-3, 2004.

Mosco, Vincent. "La economía política de las comunicaciones: Una actualización diez años después". Revista Cuadernos de Información y Comunicación, 11(2006): 57-79.

Mcchesney, R. "The political economy of communication and the future of the field". Media, cultura y sociedad. Londres: SAGE Publications, 2000.

Pérez, R. “Estructura de la radiodifusión salvadoreña". Revista La Universidad, 21(2013):171-213.

Pérez. R y Carballo. W. "El Mapa de medios en El Salvador. Todo queda en familia". Comunicación, Información y poder en El Salvador. Claves para la democratización San Salvador, El Salvador: Fundación Comunicándonos/EMA-RTV. 2013. 47-78.

Quijano, Aníbal. "Colonialidad del poder, eurocentrismo y América Latina". La colonialidad del saber: Eurocentrismo y ciencias sociales. Perspectivas Latinoamericanas. Comp. Edgardo Lander. Buenos Aires, Argentina: CLACSO, 2000. Recuperado de http://bibliotecavirtual.clacso.org.ar/ar/libros/lander/quijano.rtf

Robinson, William. Conflictos Transnacionales: Centroamérica, cambio social y globalización. San Salvador, El Salvador: UCA, 2011.

Robles, Francisco y Koen Voorend. Los dueños de la palabra en Costa Rica en un contexto de reforma neoliberal. Rupturas 1, 2(2012): 144-161.

Rockwell, Rick y Janus Noreene. Media power in Central America, Champaign, IL: University of Illinois Press, Estados Unidos, 2003.

Rothschuh, G. Estado de la Libertad de expresión en Nicaragua (2010-2013). Nicaragua: Observatorio de Medios-CINCO, 2013.

Segovia, A. Integración real y grupos de poder económico en Centroamérica: implicaciones para el desarrollo y la democracia de la región. San José, Costa Rica: Fundación Friedrich Ebert, 2005.

Thompson, J. Ideología y cultura moderna. Xochimilco, México: Universidad Autónoma Metropolitana, 2002.

José Roberto Pérez. Salvadoreño, obtuvo su título de doctor en ciencias de la comunicación en la universidad de Málaga, España, y un posgrado en economía política de la Universidad Federal de Río de Janeiro, Brasil. Es profesor de las áreas audiovisuales del departamento de periodismo de la Universidad de El Salvador, en donde actualmente es el jefe de la unidad de investigación de la Facultad de Ciencias 
y Humanidades, y miembro del concejo de investigaciones científicas, CIC-UES. Ha estudiado la estructura de medios en El Salvador con publicaciones como la estructura de la radiodifusión salvadoreña (2011), y coautor del libro Comunicación, información y poder en El Salvador: claves para la democracia (2013). También ha realizado investigaciones en economía política del sistema energético de la región con publicaciones como el precio globalizado de la electricidad: los salvadoreños como plataforma del mercado energético internacional (2015). Forma parte de las iniciativas ciudadanas que conforman el movimiento por la democratización de la comunicación.

Contacto: robperez1sv@yahoo.com

ORCID: 0000-0003-1253-9735 